\title{
The Psychometric Properties of the World Health Organization Quality of Life BREF Among Thai Women Who Have Experienced Intimate Partner Violence
}

\author{
Ratchneewan Ross, PhD, RN, FAAN \\ University of North Carolina, Greensboro, North Carolina \\ Ghada Shahrour, MSN, RN \\ Andrea Warner Stidham, PhD, RN \\ Douglas Delahanty, PhD \\ Kent State University, Ohio
}

\begin{abstract}
Background and Purpose: The World Health Organization Quality of Life BREF (WHOQOL-BREF) has been used in various populations and cultures. However, its psychometric properties are unknown among women who experienced intimate partner violence (WIPV). This study aimed to explore the reliability and validity of the WHOQOL-BREF among Thai WIPV. Methods: Two hundred eighty-four female patients receiving care at a northeast hospital in Thailand responded to the WHOQOL-BREF and intimate partner violence (IPV) questions. Exploratory factor and consistency analyses were applied. Results: Different from the original study, this study generated 5 factors, 1 of which is Medical Care Needs. The resulting scale and subscales showed good reliability and construct validity. Conclusion: The 5-factor scale can be used among WIPV by health care professionals to assess their quality of life (QOL) and appropriately address their needs.
\end{abstract}

Keywords: quality of life; intimate partner violence; psychometric properties; construct validity; World Health Organization Quality of Life BREF (WHOQOL-BREF); Thailand

$\mathrm{F}$ Tor decades, quality of life (QOL) has played a major role in health care because the concept relates to patients, clinicians, researchers, insurance companies, and regulatory agencies. Although the U.S. Food and Drug Administration may use patients' QOL as part of their decision to approve a new product or technology, clinicians and researchers can use QOL data to help them to determine the best care and interventions for their patients or participants (The Epimetrics Group, 2009; World Health Organization [WHO], 2016), including women who experience intimate partner violence (WIPV). Specifically, evidence across countries shows that individuals who experience IPV, encompassing physical, emotional, and sexual abuse, have low QOL and need corresponding professional help from nurses and other clinicians (Asadi, Mirghafourvand, Yavarikia, 
Mohammad-Alizadeh-Charandabi, \& Nikan, 2016; Breiding, Chen, \& Black, 2014; Costa et al., 2015; Ross, Stidham, Saenyakul, \& Creswell, 2015). Reliable and valid assessment of QOL is thus needed to accurately screen WIPV. This study examined the psychometric properties of the World Health Organization Quality of Life BREF (WHOQOL-BREF)/ Thai version among WIPV in Thailand.

\section{BACKGROUND AND CONCEPTUAL FRAMEWORK}

IPV (physical, sexual, and emotional) against women is a significant world health problem and a violation of human rights that occurs in various cultural, religious, and socioeconomic groups (WHO, 2013). IPV rates vary across countries, ranging from $16 \%$ in Japan to $61 \%$ in Peru for physical IPV and from $6 \%$ in Serbia to $59 \%$ in Ethiopia for sexual violence (Garcia-Moreno, Jansen, Ellsberg, Heise, \& Watts, 2006).

IPV is understudied in Thailand; yet, recent research reveals that Thailand has alarming IPV rates, ranging from 47\% to almost 90\% (Ross et al., 2015; Saito, Creedy, Cooke, \& Chaboyer, 2012). Evidence shows that IPV can negatively affect female victims physically, psychologically, and spiritually, including their overall QOL (Asadi et al., 2016; Breiding et al., 2014; Campbell, 2002; Costa et al., 2015; Kelly, 2010; Ross et al., 2015). WIPV in Thailand reported low QOL, including poor social functioning along with adverse effects on physical, psychological, and environmental health (Ross et al., 2015; Saito et al., 2012; Thananowan \& Heidrich, 2008). Ross et al. (2015) used the WHOQOL-BREF/Thai version (WHO, 2004) for the first time in Thailand to measure QOL in female patients who have experienced IPV.

Measures used to assess QOL in WIPV can vary widely because of different definitions of QOL (Lacson et al., 2010; Ross et al., 2015; Thananowan \& Heidrich, 2008). This study analyzed Ross and colleagues' (2015) existing data to examine the psychometric properties of the WHOQOL-BREF/Thai version using the WHO's definition of QOL as a guiding conceptual framework (WHO, 1996, 2004). QOL in this study is thus defined as "the individuals' perception of their position in life in the context of the culture and value systems in which they live, and in relation to their goals, expectations, standards and concerns" (WHO, 1996, p. 5).

Although the WHO's definition of QOL spans broad dimensions of life, prior research has defined QOL in a more limited manner, based mainly on depression and some physical symptoms (Thananowan \& Heidrich, 2008). For example, to assess QOL, researchers in Thailand and the United States used the 10-item Maternal Physical Health Outcome Checklist (Thananowan \& Heidrich, 2008), the Edinburgh Postnatal Depression Scale (Cox, Holden, \& Sagovsky, 1987), or the SF-12 (Lacson et al., 2010; Utah Department of Health, 2001). The SF-12 measures health-related QOL, but the WHOQOL-BREF measures both health-related and non-health-related aspects of an individual (Huang, Wu, \& Frangakis, 2006).

Non-health-related items of the WHOQOL-BREF (e.g., access to health care and social network and safety of the personal environment) are important aspects of an individual's QOL and generally valued by women in Thailand (Ross \& Ross, 2012). Furthermore, assessing QOL more broadly can inform treatment and public health decisions and provide opportunities for nurses and other clinicians to deliver personalized care to their female patients - in Thailand or elsewhere-who have experienced IPV to improve their healthrelated QOL and their QOL overall. 


\section{PROCEDURES FOR INSTRUMENT DEVELOPMENT}

The WHOQOL-BREF is an abbreviated version of the WHOQOL-100 developed by the WHO Group (WHO, 1996, 2004). The tool measures QOL beyond traditional health indicators and is not specific to Western cultures (WHO, 1996, 2004). Fifteen field centers in 15 countries including Thailand were involved in the initial development and testing of this tool. The WHOQOL-BREF has been used with various populations and cultures including female patients, adult patients with HIV/AIDS, and college students in Thailand (Li, Kay, \& Nokkaew, 2009; Ross et al., 2015; Taechaboonsermsak, Munsawaengsub, Charupoonphol, \& Charupoonphol, 2008); postpartum women and patients with chronic pain disorders in Australia (Aigner et al., 2006; Webster, Nicholas, Velacott, Cridland, \& Fawcett, 2010); adult outpatients with depression in Brazil (Berlim, Pavanello, Caldieraro, \& Fleck, 2005); patients who underwent a liver transplant in the United Kingdom (O'Carroll, Smith, Couston, Cossar, \& Hayes, 2000); and patients with cardiovascular, gastrointestinal, and respiratory problems in Korea (Min et al., 2002). The tool is available in 19 different languages such as Arabic, English, Italian, Korean, Thai, and others of African countries (WHO, 1996).

The tool has demonstrated acceptable reliability and validity in previous studies around the world. For example, across 23 countries, Skevington, Lotfy, and O'Connell (2004) reported the tool's Cronbach's alpha as $>.70$ for physical, psychological, and environmental domains and .68 for the social relations domain. In Italy, the WHOQOL-BREF/ Italian version's Cronbach's alpha ranged from .65 to .80 . The scale's construct validity was satisfactory, with a moderate correlation $(r=.40-.60)$ between the WHOQOL-BREF and MOS-SF-36 in different language versions (De Girolamo et al., 2000).

In previous studies, the WHOQOL-BREF/Thai version demonstrated a good Cronbach's alpha of .83 in adult patients with HIV/AIDS and .86 in college students (Li et al., 2009; Taechaboonsermsak et al., 2008). To the best of our knowledge, no previous studies have examined other psychometric properties of this measure in Thailand, let alone among WIPV.

\section{METHODS}

\section{Design}

This article is part of a larger convergent mixed methods study that used structural equation modeling and qualitative description to examine and describe the association between IPV, emotional support, and health outcomes among Thai women (Ross et al., 2015). This study assessed the psychometric properties of the WHOQOL-BREF/Thai version using exploratory factor analysis, convergent and discriminant validity analysis, and reliability analysis.

\section{Sample}

The sample included 284 Thai women who had experienced varying types of IPV: $22.2 \%$ experienced all three types of IPV, $41.9 \%$ experienced two types, $26.1 \%$ experienced one type, and $9.1 \%$ experienced no IPV. The sample size meets the requirement of 10 subjects per item for conducting psychometric analyses (Hair, Black, Babin, \& Anderson, 2010; Kline, 2011). Eligible participants were adult female patients ( $\geq 18$ years old) receiving obstetrician/gynecologist $(\mathrm{OB} / \mathrm{GYN})$ care in several in- and outpatient units at a large hospital in Northeast Thailand. 


\section{Data Collection Procedure}

Data collection in the parent study took place between July and October of 2010. Two internal review boards in the United States and Thailand approved the study protocol. The principal investigator of the study trained registered nurses (RNs) on how to collect data for the project, starting with their explanation of the study's objectives, benefits, and risks, followed by a verbal consent. Following consent, participants completed a structured selfreport questionnaire packet in a private room. RNs were always present in case any participants became distressed during completion of study materials; none of the participants showed any distress symptoms.

\section{Instruments}

WHOQOL-BREF/Thai version (WHO, 1996, 2013) includes 26 questions, 24 of which assess four dimensions: physical health, psychological health, environmental health, and social relations (WHO, 1996). Two additional items assessing the overall QOL and general health are not considered under any of the four dimensions (WHO, 1996, 2013) and were thus excluded from this study for factor analysis. The WHOQOL-BREF is a Likert-type questionnaire asking the participant to rate her perception of specific QOL in the past 2 weeks. Potential answers range from not at all (1) to completely (5). An example question is "How satisfied are you with your sex life?" Three items out of 26 are negatively worded. Thus, these items' scores are reversed before the final calculation. The higher the total score, the higher the level of QOL as perceived by the participant.

The Thai Depression Inventory (TDI; Lotrakul \& Sukanich, 1999) is a 20-item, self-report instrument measuring the severity of depression in the past week. For each of the 20 items, the participant is instructed to choose the statement (out of four) that best describes her moods or thoughts, ranging from no symptoms (1) to most severe (4). For example, (1) I never think of death, (2) I think of death often but don't want to die, (3) I want to die, and (4) I am planning to end my life. The Cronbach's alpha in this study was .86. Higher total scores indicate more depression. The tool's construct validity was supported during the tool development by its association with the Hamilton Rating Scale for Depression ( $r=.72$; Lotrakul \& Sukanich, 1999).

\section{Data Analysis}

In the larger study, data were doubled check for entering accuracy and cleaned using SPSS Version 21. Less than $2 \%$ of the data were missing; thus, an item mean was used to replace missing values. Exploratory factor analysis and internal consistency were analyzed. Pearson's $r$ was performed to explore the association between the WHOQOLBREF/Thai version and the TDI. Also, one-way analysis of variance (ANOVA) was used to compare QOL composite scores among four subgroups of participants with differing IPV experiences.

\section{RESULTS}

Participants ranged in age from 18 to 58 years old. Approximately 94\% were married/ living with a partner and $20 \%$ were pregnant. About half of the participants had completed at least some college and worked at a professional level. Almost $90 \%$ of participants reported emotional violence, more than $61 \%$ physical violence, and $25 \%$ sexual violence. 


\section{Construct Validity}

In this study, construct validity of the tool was examined for its dimensionality, convergent validity, and discriminant validity. Details are described in the following text.

Dimensionality. Results indicated factorability of the scale: $44 \%$ of the correlations among items were at least .30; the Kaiser-Meyer-Olkin (KMO) value for sample adequacy was .87, and the Bartlett's test of sphericity was significant $(p<.001$; Hair et al., 2010). Next, principal component analysis was performed to extract the minimum number of factors that explain the maximum variance for the scale using the criteria of (a) eigenvalue of $>1$, (b) factor explained variance percentage of $\geq 5 \%$, and (c) accumulative explained variance percentage of $\geq 50 \%$ ( $56.8 \%$ in this study). Based on the team's careful examination of the nature and meaning of items in each emergent cluster, we named these factors as follows: Physical Health, Self and Spirituality, Psychological Health and Relationship, Safety and Environment, and Medical Care Needs. Subsequently, varimax rotation was performed with a suppression of loadings $<.40$. Results revealed that 19 out of 24 items loaded cleanly and strongly on one of the resulting five factors (Table 1). Table 2 compares the resulting factors to the WHO's factors.

Convergent Validity. Convergent validity was supported by a significant moderate negative correlation between the WHOQOL-BREF/Thai version and the TDI (Lotrakul \& Sukanich, 1999), indicating that the lower the QOL, the more depression the Thai women experienced $(r=-.55, p<.01)$.

Discriminant Validity. In our study, the tool was further validated by comparing the mean scores among four subgroups of participants who experienced differing degrees of IPV: no IPV, one type of IPV, two types to IPV, and all three types of IPV. Theoretically, women who have not experienced IPV are expected to have the best QOL, followed by those with one, two, and three types of IPV. Indeed, in our study, one-way ANOVA results resonate with the theoretical speculation, $F=24.5, d f(280,3), p<.001$ (Figure 1). Bonferroni post hoc analysis showed that the composite QOL scores were statistically significant between all six pairs (see Figure 1), thus supporting the theoretical assumption and discriminant validity of the WHOQOL-BREF.

\section{Reliability}

The WHOQOL-BREF/Thai version's overall Cronbach's alpha in our study was .87, exceeding the minimum acceptable criterion of .70 (Hair et al., 2010). Deletion of any items would not increase the scale's internal consistency. Most of the item-to-total scale correlations $(92.3 \%)$ were within the acceptable range $(r=.30-.70$; Hair et al., 2010). The five subscales generated good Cronbach's alphas ranging from .74 to .80 (see Table 2).

\section{DISCUSSION AND LIMITATIONS}

Findings from our study have added new knowledge concerning the WHOQOL-BREF/ Thai version (WHO, 1996, 2013) as a measure of QOL. Results supported the reliability and validity of the tool. Using exploratory factor analysis, two questionnaire itemsphysical pain and dependence on medical treatment-had the strongest loadings and loaded strongly $(>.70)$ on the Medical Care Needs factor, suggesting that these items are a major part of QOL in this sample of Thai women. As most of our participants (almost 
TABLE 1. Results of Principal Component Analysis With Varimax Rotation

\begin{tabular}{|c|c|c|c|c|c|}
\hline \multirow[b]{2}{*}{ Item } & \multicolumn{5}{|c|}{ Component } \\
\hline & 1 & 2 & 3 & 4 & 5 \\
\hline $\begin{array}{l}\text { 3. To what extent do you feel that physical pain } \\
\text { prevents you from doing what you need to do? }\end{array}$ & & & & & .869 \\
\hline $\begin{array}{l}\text { 4. How much do you need any medical treatment to } \\
\text { function in your daily life? }\end{array}$ & & & & & .858 \\
\hline 5. How much do you enjoy life? & & & .495 & & \\
\hline $\begin{array}{l}\text { 6. To what extent do you feel your life to be } \\
\text { meaningful? }\end{array}$ & & & .660 & & \\
\hline 7. How well are you able to concentrate? & & & .708 & & \\
\hline 8. How safe do you feel in your daily life? & & .560 & .487 & & \\
\hline 9. How healthy is your physical environment? & & 640 & & & \\
\hline 10. Do you have enough energy for everyday life? & .484 & & .541 & & \\
\hline 11. Are you able to accept your bodily appearance? & & & .565 & & \\
\hline 12. Have you enough money to meet your needs? & & .700 & & & \\
\hline $\begin{array}{l}\text { 13. How available to you is the information that you } \\
\text { need in your day-to-day life? }\end{array}$ & & 679 & & & \\
\hline $\begin{array}{l}\text { 14. To what extent do you have the opportunity for } \\
\text { leisure activities? }\end{array}$ & & .588 & & & \\
\hline 15. How well are you able to get around? & .440 & & & & \\
\hline 16. How satisfied are you with your sleep? & .563 & & & & \\
\hline $\begin{array}{l}\text { 17. How satisfied are you with your ability to } \\
\text { perform your daily living activities? }\end{array}$ & .683 & & & & \\
\hline 18. How satisfied are you with your capacity for work? & .609 & & & .474 & \\
\hline 19. How satisfied are you with yourself? & .452 & & & .593 & \\
\hline $\begin{array}{l}\text { 20. How satisfied are you with your personal } \\
\text { relationships? }\end{array}$ & & & & 689 & \\
\hline 21. How satisfied are you with your sex life? & & & & .691 & \\
\hline $\begin{array}{l}\text { 22. How satisfied are you with the support you get } \\
\text { from your friends? }\end{array}$ & & & & .653 & \\
\hline $\begin{array}{l}\text { 23. How satisfied are you with the conditions of your } \\
\text { living place? }\end{array}$ & .436 & & & & \\
\hline $\begin{array}{l}\text { 24. How satisfied are you with your access to health } \\
\text { services? }\end{array}$ & .480 & .469 & & & \\
\hline 25. How satisfied are you with your transport? & .647 & & & & \\
\hline $\begin{array}{l}\text { 26. How often do you have negative feelings such as } \\
\text { blue mood, despair, anxiety, depression? }\end{array}$ & & & & .431 & \\
\hline
\end{tabular}

Note. Loadings were suppressed if they were $<.40$. Cross loading items were assigned to the factor they loaded more heavily on. 
TABLE 2. Comparison of Factors and Alphas Resulting From the World Health Organization Original Study and the Present Study (No. of Items = 24)

\begin{tabular}{|c|c|c|c|c|}
\hline \multirow[b]{2}{*}{ No. } & \multicolumn{2}{|l|}{ WHO's Findings } & \multicolumn{2}{|l|}{ Present Study's Findings } \\
\hline & Factor & Alpha & Factor & Alpha \\
\hline \multirow[t]{8}{*}{1} & Physical health (7 items): & .80 & Physical health (7 items): & .79 \\
\hline & 3. Physical pain & & 15. Getting around & \\
\hline & 4. Medical attention needs & & 16. Sleep & \\
\hline & 10. Energy & & 17. Daily living activities & \\
\hline & 15. Getting around & & 18. Work capacity & \\
\hline & 16. Sleep & & 23. Living conditions & \\
\hline & 17. Daily living activities & & 24. Access to health care & \\
\hline & 18. Work capacity & & 25. Transportation & \\
\hline \multirow[t]{6}{*}{2} & Psychological health (6 items): & .76 & $\begin{array}{l}\text { Psychological health and } \\
\text { relationship (5 items): }\end{array}$ & .74 \\
\hline & 6 Meaninoful life & & 19. Satisfaction with self & \\
\hline & 7. Concentration & & 20. Personal relationship & \\
\hline & 11. Body appearance & & 21. Sex life & \\
\hline & 19. Satisfaction with self & & 22. Friend support & \\
\hline & $\begin{array}{l}\text { 26. Despair, anxiety, } \\
\text { depression }\end{array}$ & & $\begin{array}{l}\text { 26. Despair, anxiety, } \\
\text { depression }\end{array}$ & \\
\hline \multirow[t]{6}{*}{3} & Social relationships (3 items): & .66 & Self and spirituality (5 items): & .75 \\
\hline & 20. Personal relationship & & 5. Life enjoyment & \\
\hline & 21. Sex life & & 6. Meaningful life & \\
\hline & 22. Friend support & & 7. Concentration & \\
\hline & & & 10. Energy & \\
\hline & & & 11. Body appearance & \\
\hline \multirow[t]{9}{*}{4} & Environment (8 items): & .80 & Safety and environment (5 items): & .80 \\
\hline & 8. Safety & & 8. Safety & \\
\hline & 9. Physical environment & & 9. Physical environment & \\
\hline & 12. Finance & & 12. Finance & \\
\hline & 13. Information & & 13. Information & \\
\hline & 14. Leisure activities & & 14. Leisure activities & \\
\hline & 23. Living conditions & & & \\
\hline & 24. Access to health care & & & \\
\hline & 25. Transportation & & & \\
\hline \multirow[t]{3}{*}{5} & NA & NA & Medical care needs (2 items): & .77 \\
\hline & & & 3. Physical pain & \\
\hline & & & 4. Medical attention needs & \\
\hline
\end{tabular}

Note. $\mathrm{WHO}=$ World Health Organization; NA = not applicable. 


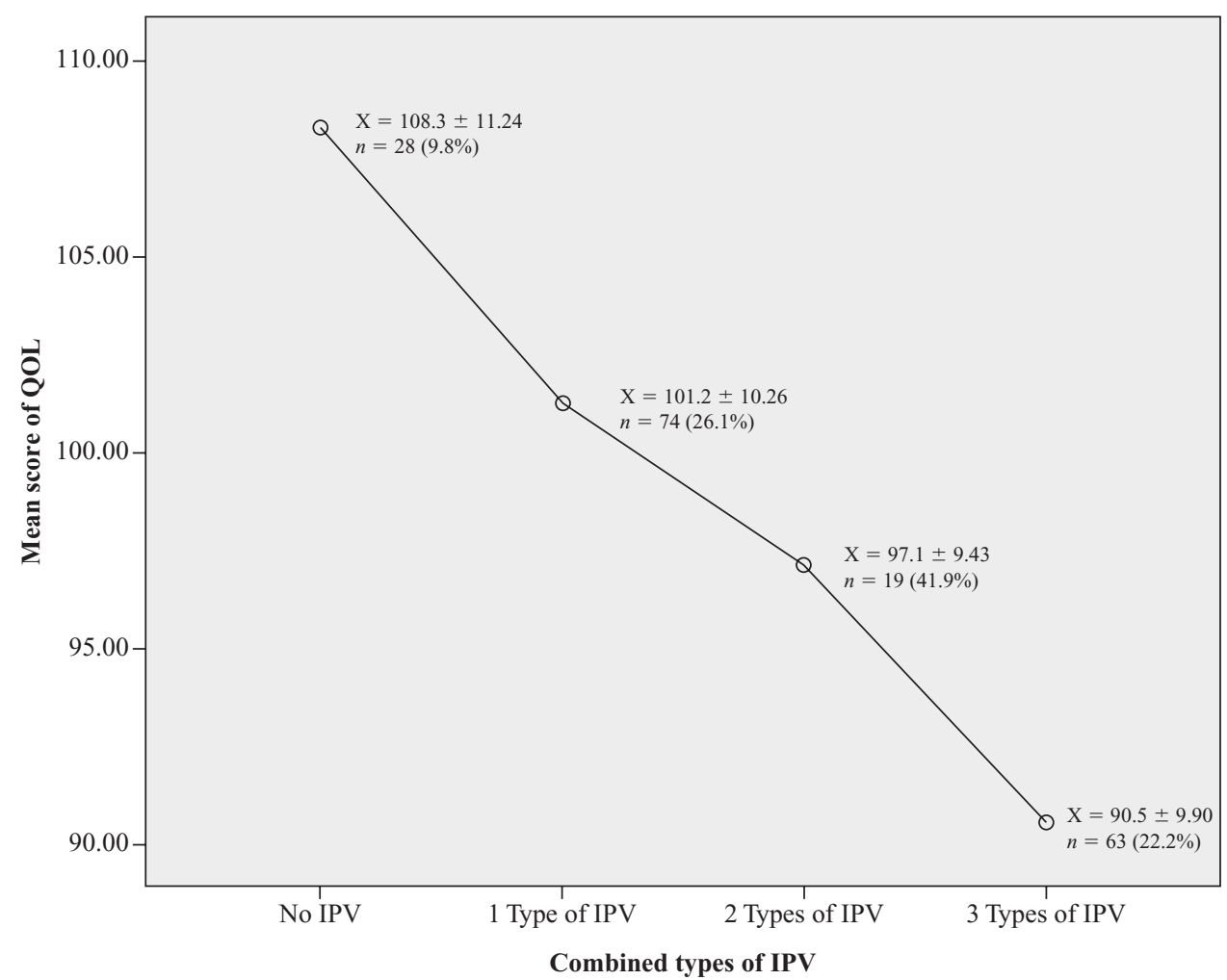

\begin{tabular}{|lccc|}
\hline Bonferroni: & & & \\
\hline Groups & Mean Difference & $S E$ & $p$ Value \\
\hline No IPV versus 1 type of IPV & 7.1 & 2.21 & $<.05$ \\
No IPV versus 2 types of IPV & 11.2 & 2.09 & $<.001$ \\
No IPV versus 3 types of IPV & 17.8 & 2.27 & $<.001$ \\
1 type of IPV versus 2 types of IPV & 4.1 & 1.48 & $<.05$ \\
1 type of IPV versus 3 types of IPV & 10.7 & 1.71 & $<.001$ \\
2 types of IPV versus 3 types of IPV & 6.6 & 1.55 & $<.001$ \\
\hline
\end{tabular}

Figure 1. Quality of life (QOL) mean score comparisons among four subgroups of Thai women with combined types of intimate partner violence (IPV; $N=284$ ).

90\%) had experienced at least one type of IPV, most of which was physical violence (>61\%; Ross et al., 2015), it is not surprising that they valued QOL highest on their medical care needs.

Overall, the tool yielded five factors as opposed to the four factors originally reported by WHO (1996, 2013; see Table 2). The differences can be explained in that the WHOQOLBREF/Thai version was tested in Thailand in a different population from this study. The previous study tested the tool in Bangkok, the capital city of Thailand, among individuals 
ages 12-97 years (half were males, $17 \%$ were healthy, and $83 \%$ had various diseases and disorders), whereas this study used the tool to measure QOL in Northeast Thailand among Thai women ages 18-58 years who had different IPV experiences. These differing regions and populations could have accounted for the different loading results between these two Thai studies (Ross et al., 2015; WHO, 1996, 2013).

The construct validity of the WHOQOL-BREF/Thai version was further confirmed through its significant negative correlation with depression (using the TDI). This finding is in line with previous studies, which also found the WHOQOL-BREF to be negatively associated with other measures of depression (Berlim et al., 2005; Naumann \& Byrne, 2004).

The WHOQOL-BREF/Thai version in our study generated good Cronbach's alphas for both the total scale and subscales and is comparable to the original scale Cronbach's alphas. Our findings are consistent with previous studies in other Thai populations ( $\mathrm{Li}$ et al., 2009; Taechaboonsermsak et al., 2008), which reported acceptable reliability of the scale $(\alpha>$.70).

The WHOQOL-BREF can be used with confidence in Thailand among female patients who have experienced varying types of IPV. To identify QOL needs, nurses and other health care professionals should administer the tool to Thai female patients using five factors so that their physical health, psychological health and relationship, self and spirituality, safety and environment, and especially medical care needs can be examined and appropriately addressed.

Our results are specific to Thai WIPV who used health care in Northeast Thailand, thus limiting generalizability to other female groups in Thailand and other cultures. Another limitation is that test-retest reliability could not be reported in this study because the data were collected cross-sectionally. Further longitudinal assessment of the psychometric properties of the WHOQOL-BREF/Thai version in other female populations in Thailand and in WIPV in other cultures is recommended.

\section{NURSING IMPLICATIONS AND CONCLUSION}

Despite these limitations, the present results underscore the validity of the WHOQOLBREF/Thai version and suggest the utility of the tool as a reliable screening measure for these high-risk women. Nurses and other health care professionals can incorporate this simple and short screener into their standard care practices to allow for the efficient identification of problematic QOL. Nursing educators can also include the tool in the classroom and clinical setting when teaching their students to evaluate QOL, especially in the target population. Finally, the WHOQOL-BREF/Thai version can be used in future research to measure QOL among Thai WIPV in Thailand and other countries.

\section{REFERENCES}

Aigner, M., Förster-Streffleur, S., Prause, W., Freidl, M., Weiss, M., \& Bach, M. (2006). What does the WHOQOL-Bref measure? Measurement overlap between quality of life and depressive symptomatology in chronic somatoform pain disorder. Social Psychiatry and Psychiatric Epidemiology, 41, 81-86. http://dx.doi.org/10.1007/s00127-005-0997-8

Asadi, S., Mirghafourvand, M., Yavarikia, P., Mohammad-Alizadeh-Charandabi, S., \& Nikan, F. (2016). Domestic violence and its relationship with quality of life in Iranian women of reproductive age. Journal of Family Violence, 32, 1-8. http://dx.doi.org/10.1007/s10896-016-9832-0 
Berlim, M. T., Pavanello, D. P., Caldieraro, M. A., \& Fleck, M. P. (2005). Reliability and validity of the WHOQOL BREF in a sample of Brazilian outpatients with major depression. Quality of Life Research, 14, 561-564.

Breiding, M. J., Chen, J., \& Black, M. C. (2014). Intimate partner violence in the United States-2010. Atlanta, GA: National Center for Injury Prevention and Control, Centers for Disease Control and Prevention.

Campbell, J. C. (2002). Heath consequences of intimate partner violence. Lancet, 359, 1331-1336.

Costa, D., Hatzidimitriadou, E., Ioannidi-Kapolou, E., Lindert, J., Soares, J., Sundin, Ö., . . . Barros, H. (2015). Intimate partner violence and health-related quality of life in European men and women: Findings from the DOVE study. Quality of Life Research, 24(2), 463-471. http:// dx.doi.org/10.1007/s11136-014-0766-9

Cox, J. L., Holden, J. M., \& Sagovsky, R. (1987). Detection of postnatal depression: Development of the 10-item Edinburgh Postnatal Depression Scale. The British Journal of Psychiatry, 150, $782-786$.

De Girolamo, G., Rucci, P., Scocco, P., Becchi, A., Coppa, F., D’Addario, A., . . Soldani, L. (2000). Quality of life assessment: Validation of the Italian version of the WHOQOL-Brief. Epidemiologia e Psichiatria Sociale, 9(1), 45-55.

Garcia-Moreno, C., Jansen, H., Ellsberg, M., Heise, L., \& Watts, C. (2006). Prevalence of intimate partner violence: Findings from the WHO multi-country study on women's health and domestic violence. Lancet, 368, 1260-1269.

Hair, J. F., Black, W. C., Babin, B. J., \& Anderson, R. E. (2010). Multivariate data analysis. Upper Saddle River, NJ: Prentice Hall.

Huang, I. C., Wu, A. W., \& Frangakis, C. (2006). Do the SF-36 and WHOQOL-BREF measure the same constructs? Evidence from the Taiwan population. Quality of Life Research, 15, 15-24. http://dx.doi.org/10.1007/s11136-005-8486-9

Kelly, U. (2010). Intimate partner violence, physical health, posttraumatic stress disorder, depression, and quality of life in Latinas. The Western Journal of Emergency Medicine, 11(3), 247-251.

Kline, R. B. (2011). Principles and practice of structural equation modeling (3rd ed.). New York, NY: Guilford Press.

Lacson, E., Xu, J., Lin, S., Dean, S. G., Lazarus, M., \& Hakim, R. M. (2010). A comparison of SF-36 and SF-12 composite scores and subsequent hospitalization and mortality risks in long-term dialysis patients. Clinical Journal of the American Society of Nephrology, 5, 252-260.

Li, K., Kay, N. S., \& Nokkaew, N. (2009). The performance of the World Health Organization's WHOQOL-BREF in assessing the quality of life of Thai college students. Social Indicators Research, 90(3), 489-501.

Lotrakul, M., \& Sukanich, P. (1999). Development of the Thai Depression Inventory. Journal of the Medical Association of Thailand, 82(12), 1200-1207.

Min, S. K., Kim, K. I., Lee, C. I., Jung, Y. C., Suh, S. Y., \& Kim, D. K. (2002). Development of the Korean versions of WHO Quality of Life scale and WHOQOL-BREF. Quality of Life Research, $11,593-600$.

Naumann, V. J., \& Byrne, G. J. (2004). WHOQOL-BREF as a measure of quality of life in older patients with depression. International Psychogeriatrics, 16(2), 159-173.

O'Carroll, R. E., Smith, K., Couston, M., Cossar, J. A., \& Hayes, P. C. (2000). A comparison of the WHOQOL-100 and the WHOQOL-BREF in detecting change in quality of life following liver transplantation. Quality of Life Research, 9, 121-124.

Ross, R., \& Ross, J. (2012). People of Thai heritage. In L. D. Purnell (Ed.), Transcultural health care: A culturally competent approach (4th ed.). Philadelphia, PA: F. A. Davis. Retrieved from http://resources.fadavis.com/purnell4e/bonus_chapters/Ch36_001-018.pdf

Ross, R., Stidham, A. W., Saenyakul, P., \& Creswell, J. (2015). Intimate partner violence, emotional support, and health outcomes among Thai women: A mixed methods study. Journal of the Royal Thai Army Nurses, 16(1), 14-24.

Saito, A., Creedy, D., Cooke, M., \& Chaboyer, W. (2012). Effect of intimate partner violence on postpartum women's health in Northeastern Thailand. Nursing \& Health Sciences, 14, 345-351.

Skevington, S. M., Lotfy, M., \& O'Connell, K. A. (2004). The World Health Organization's WHOQOL-BREF quality of life assessment: Psychometric properties and results of the international field trial: A report from the WHOQOL group. Quality of Life Research, 13(2), 299-310. 
Taechaboonsermsak, P., Munsawaengsub, C., Charupoonphol, S., \& Charupoonphol, P. (2008). Quality of life of HIV/AIDS patients in Bangkok Metropolitan. Journal of Public Health, 91-103.

Thananowan, N., \& Heidrich, S. M. (2008). Intimate partner violence among pregnant Thai women. Violence Against Women, 14(5), 509-527.

The Epimetrics Group. (2009). The importance of quality-of-life research. Retrieved from http:// myhealthoutcomes.com/pages/3000

Utah Department of Health. (2001). Interpreting the SF-12. Retrieved from http://health.utah.gov/ opha/publications/2001hss/sf12/SF12_Interpreting.pdf

Webster, J., Nicholas, C., Velacott, C., Cridland, N., \& Fawcett, L. (2010). Validation of the WHOQOL-BREF among women following childbirth. The Australian \& New Zealand Journal of Obstetrics \& Gynaecology, 50(2), 132-137.

World Health Organization. (1996). WHOQOL-BREF. Introduction, administration, scoring and generic version of the assessment. Retrieved from http://www.who.int/mental_health/media/ en/76.pdf

World Health Organization. (2004). Intimate partner violence. Geneva, Switzerland: Author.

World Health Organization. (2013). Global and regional estimates of violence against women: Prevalence and health effects of intimate partner violence and non-partner sexual violence. Retrieved from http://apps.who.int/iris/bitstream/10665/85239/1/9789241564625_eng.pdf

World Health Organization. (2016). WHOQOL: Measuring quality of life. Retrieved from http:// www.who.int/healthinfo/survey/whoqol-qualityoflife/en/

Acknowledgments. The original study was supported by the Fulbright Foundation to first author (RR).

Correspondence regarding this article should be directed to Ratchneewan Ross, PhD, RN, FAAN, School of Nursing, University of North Carolina at Greensboro, 1 Petty Building, McIver St., Greensboro, NC 27402. E-mail: r_ross2@uncg.edu 